\title{
Electrochemical STM study of the adsorption of adenine on $\mathrm{Au}(111)$ electrodes
}

\author{
Cristina Vaz-Domínguez a , María Escudero-Escribano a,1, Angel Cuesta ${ }^{\mathrm{a}, 2}$, Francisco Prieto-Dapena ${ }^{\mathrm{b}, *}$, \\ Consuelo Cerrillos ${ }^{c}$, Manuela Rueda ${ }^{\mathrm{b}}$ \\ a Instituto de Química Física “Rocasolano” CSIC, C. Serrano 119, E-28006 Madrid, Spain \\ b Department of Physical Chemistry, Faculty of Pharmacy, University of Seville, c/Profesor García González 2, Sevilla E-41012, Spain \\ c Centre for Research, Technology and Innovation, University of Seville, Av. Reina Mercedes 4, Sevilla E-41012, Spain
}

\section{A R T I C L E I N F O}

\section{Article history:}

Received 5 July 2013

Received in revised form 27 July 2013

Accepted 29 July 2013

Available online 11 August 2013

\section{Keywords:}

Adenine adsorption

$\mathrm{Au}(111)$ electrodes

Electrochemical scanning tunneling

microscopy

Surface reconstruction

$\pi$-Stacking

\begin{abstract}
A B S T R A C T
An electrochemical scanning tunneling microscopy (EC-STM) study of the adsorption of adenine on $\mathrm{Au}(111)$ electrodes was undertaken in the $\mathrm{pH}$ range between 1 and 7, aiming at achieving a deeper knowledge on the structure and organization of adenine chemisorbed on gold and at confirming previous conclusions obtained from combined electrochemical and in situ IR spectroscopy measurements. This study confirms that chemisorption of adenine induces the lifting of the $\mathrm{Au}(111)$ surface reconstruction. Furthermore, the $4 \%$ excess gold atoms of the reconstructed surface, which are expelled during lifting of the reconstruction, do not diffuse away from the reconstruction rows. We observe, in contrast, the formation of nanometric islands arranged forming chains along the directions previously followed by the reconstruction solitons. Chemisorbed adenine adlayers consist of short chains of adenine molecules roughly aligned along the three main crystallographic directions of the substrate and stabilized by $\pi$ stacking. These chains tend to align parallel to each other, forming very small domains and yielding an adlayer with a very short-range order. The same adlayer structure is observed at all the studied $\mathrm{pH}$ values. The STM results also confirm that, in very acidic media, and at low potentials, adenine adsorbs very weakly on the reconstructed $\mathrm{Au}(111)$ surface.
\end{abstract}

(c) 2013 Elsevier B.V. All rights reserved.

\section{Introduction}

The study of the adsorption of DNA bases on well-defined electrode surfaces bears interest due to their biological relevance and to the technical importance of the preparation of biocompatible materials and biosensors. In this context, we have recently focused our studies on the adsorption of adenine on gold single-crystal and thin-film electrodes [1-4].

Initially, we characterised the adsorption of adenine on low-index gold single-crystal electrodes by cyclic voltammetry and impedance measurements [1]. Adenine adsorption showed a significant sensitivity to the reconstructed or unreconstructed state of the electrode surface. Chemisorption of adenine was found to lift the reconstruction on all three low-index surfaces, and the thermodynamic study of the adsorption/desorption of adenine on Au (111) surfaces by means of chronocoulometry established that the chemisorption is weak and that adenine behaves as an electron donor [1]. The thermodynamic

\footnotetext{
* Corresponding author. Tel.: + 34954556734.

E-mail address: dapena@us.es (F. Prieto-Dapena).

1 Present address: Center of Individual Nanoparticle Functionality, Department of Physics, Technical University of Denmark, DK-2800 Kongens Lyngby, Denmark.

2 Present address: Department of Chemistry, School of Natural and Computing Sciences, University of Aberdeen, Aberdeen AB24 3UE, UK
}

analysis also provided a projected area of the adsorbed molecule of $44 \AA^{2}$, this being compatible with a tilted orientation of the molecular plane relative to the electrode surface. A study of the kinetics of the adsorption process using electrochemical impedance spectroscopy [2] revealed a mixed control of the adsorption rate by both mass transport and activation steps.

In order to obtain information about the microscopic aspects of the adsorption process, in situ IR spectroscopy was applied [3], both in the external reflection-absorption (IRRAS) configuration with Au (111) electrodes, and in the attenuated total reflectionsurface-enhanced infrared absorption (ATR-SEIRAS) configuration with nanostructured gold thin-film electrodes. The assignments of the surface-active IR signals and the analysis of their potential dependence confirmed a tilted orientation of the adsorbed adenine molecular plane relative to the electrode surface, and that the molecular orientation does not change with the electrode potential, respectively.

The influence of the $\mathrm{pH}$ on the IR spectra of adsorbed adenine [4] allowed us to conclude that adenine adsorbs in its neutral (unprotonated) form even at pHs below its $\mathrm{p} K_{1}$ (4.2). On the other hand, in very acidic media and at low adenine concentrations, ATR-SEIRAS revealed the existence, at potentials below the onset of the chemisorption of adenine, of a second form of adsorbed adenine, which was identified as physisorbed protonated adenine. IR spectra also suggested the coadsorption of water in acidic media. 
The structure of adenine adlayers on solid substrates strongly depends on the chemical nature of the substrate [5-9]. A combined STM and electron energy loss spectroscopy (EELS) study in ultrahigh vacuum (UHV) revealed that adenine adsorbs on $\mathrm{Cu}(110)$ with the molecular plane parallel to the substrate with a tilted $\mathrm{C}-\mathrm{NH}_{2}$ bond, forming chains that order themselves into chiral domains linked by H-bonding interactions [5]. On graphite electrodes [6-8], adsorbed adenine forms incommensurate condensed layers with a structure strongly dependent on the applied potential. The STM images obtained for the adsorption of adenine on $\mathrm{Au}(111)$ electrodes at potentials around the open circuit potential [9] suggest a different organisation, with adenine molecules aligned along the main crystallographic directions, and with intermolecular distances clearly shorter than those corresponding to hydrogen bonding interactions.

The above-mentioned recent information about adenine adsorption on $\mathrm{Au}$ (111) electrodes provided by combining electrochemical methods with in situ IR spectroscopy has induced us to revisit the problem using STM, which can help to clarify the role played by the $\mathrm{pH}$, the potential, the reconstructed or unreconstructed state of the surface and the nature of the adenine-adenine interactions. We report here an electrochemical STM study of the adsorption of adenine on $\mathrm{Au}(111)$ electrodes as a function of the electrode potential in the $\mathrm{pH}$ range between 1 and 7. Structural information about the arrangement of adenine molecules within the adlayer and about the nature of the corresponding intermolecular interactions is provided. The adlayer structure is found to be potential-independent within the chemisorption region. Moreover, the role of adsorbed adenine in the surface reconstruction phenomenon is clarified.

\section{Materials and methods}

A Pico LE and a Pico Plus Molecular Imaging microscopes with PicoScan 2100 and 2500 controllers, respectively, were employed. Tungsten tips were electrochemically etched in $2 \mathrm{M} \mathrm{NaOH}$ from a wire $0.25 \mathrm{~mm}$ in diameter and were then covered either with an electrophoretic lack or with polyethylene based thermal glue (ethyl-vinyl-acetate) from a polyethylene glue gun. All the images were obtained in the constant-current mode with currents lower than $2 \mathrm{nA}$ because, at higher tunnelling currents, we often observed that the structure of the adenine adlayer was destroyed by the STM tip during scanning.

The electrochemical STM cell was fabricated in PCTFE (Kel-F), with a capacity of ca. $100 \mu \mathrm{L}$. It was left overnight in "piranha" solution and thoroughly rinsed with ultrapure boiling water before each measurement. The working electrode was a $\mathrm{Au}(111)$ disk $12 \mathrm{~mm}$ in diameter and $2 \mathrm{~mm}$ thick from MaTecK (Jülich, Germany), which was flame annealed before each experiment. A platinum wire was used as pseudo-reference electrode, but all the potentials in the text are referred to the saturated calomel electrode (SCE). A gold wire was used as the auxiliary electrode.

Working solutions with $\mathrm{pH}$ between 1 and $7\left(0.1 \mathrm{M}\right.$ in $\mathrm{HClO}_{4}$, $0.1 \mathrm{M} \mathrm{H}_{2} \mathrm{SO}_{4}, 0.1 \mathrm{M} \mathrm{KClO}_{4}$ and $\left.(0.1-x) \mathrm{M} \mathrm{HClO}_{4}+x \mathrm{M} \mathrm{KClO}_{4}\right)$ containing $1 \mathrm{mM}$ adenine were prepared with ultrapure Milli-Q water ( $18 \mathrm{M} \Omega \mathrm{cm},<3 \mathrm{ppb}$ TOC), $70-72 \% \mathrm{HClO}_{4}$ (Merck, p.a.), $96 \%$ $\mathrm{H}_{2} \mathrm{SO}_{4}$ (Merck Suprapur), 99\% $\mathrm{KClO}_{4}$ (Merck, p.a.) and $99 \%$ adenine (Fluka, p.a.). The working electrode was put in contact with the adenine-containing solution under potential control, negative of the onset of the lifting of the reconstruction, in order to avoid the lifting of the thermally induced reconstruction formed during flame annealing [10].

\section{Results and discussion}

Fig. 1 shows the cyclic voltammogram of $\mathrm{Au}(111)$ in $0.1 \mathrm{M}$ $\mathrm{H}_{2} \mathrm{SO}_{4}+1 \mathrm{mM}$ adenine and a sequence of STM images obtained in the same solution at different electrode potentials in a separate experiment. Similar results were obtained in all the solutions used. The
STM experiment was started with a freshly flame annealed electrode at the negative potential limit, and the potential was then stepped to successively higher values. At potentials lower than the onset of chemisorption of adenine, only the herringbone structure characteristic of the thermally induced reconstruction can be observed (Fig. 1a and b). At these conditions, physisorbed protonated adenine has been detected on the $\mathrm{Au}(111)$ surface by ATR-SEIRAS [4]. The persistence of the reconstructed surface confirms that the interaction of protonated adenine with the electrode surface at these potentials is very weak. The failure to image physisorbed adenine with the STM might be due to adenine molecules being swept away from the electrode surface by the tip during scanning or, alternatively, to a high mobility of the adenine molecules in the physisorbed adlayer.

As the potential increases, the reconstruction rows first become sharper and finally disappear, as can be observed in Fig. 1b and c. These changes have been recently shown to be characteristic of the transition from the reconstructed to the unreconstructed surface in a detailed study in several supporting electrolytes of the initial stages of the lifting of the surface reconstruction on $\mathrm{Au}(111)$ electrodes [11], that was shown to occur always through the same sequence of dislocation networks. It was also shown there that, independently of the nature and concentration of the supporting electrolyte, the lifting of the reconstruction process starts always at a surface charge density of $-5 \mu \mathrm{C} \mathrm{cm}^{-2}$ and concludes at a surface charge density of $28 \mu \mathrm{C} \mathrm{cm}^{-2}$, when the dislocations disappear and the surface reaches its unreconstructed state. Similar morphologies, but in the reverse process, have been recently observed by He and Borguet [12]. It must be noted, however, that Wu et al. [14] have demonstrated that, at similar charge densities, the adsorption of some organic molecules on the unreconstructed surface is thermodynamically more favourable and, therefore, has a significant contribution to the driving force of the lifting of the reconstruction.

Concomitantly with the lifting of the reconstruction, gold islands with a diameter between 2 and $10 \mathrm{~nm}$ and of monotatomic height appear on the surface in order to accommodate the $4 \%$ excess atoms of the reconstruction (Fig. 1d-f). Contrary to the behaviour in adenine-free sulphuric acid or perchloric acid/perchlorate solutions, in which islands grow by Ostwald ripening and/or diffuse to the edge of neighbouring terraces, in the presence of adenine they remain frozen at the positions where the atoms were ejected, and decorate the surface by aligning themselves along the directions previously occupied by the reconstruction lines (Fig. 2a). These results are similar to those reported by Dretschkow et al. [13] in a study of the adsorption of uracil on $\mathrm{Au}(111)$ electrodes. These authors found that, by applying a sufficiently negative potential for at least $60 \mathrm{~min}$, the electrochemically induced reconstruction forms, but the resulting morphology has a shorter-range order than the thermally induced reconstruction formed during flame annealing [13]. The observed freezing of the gold islands at the locations where they are initially formed and the slow formation of the electrochemically induced surface reconstruction are both a consequence of the adsorbate-induced low mobility of gold surface atoms, in agreement with the conclusions reached by Wu et al. concerning the adsorption of other organic molecules [14].

On the unreconstructed $\mathrm{Au}(111)$ surface, adenine chemisorbs forming short chains that tend to align parallel to each other, and that roughly follow the main crystallographic directions of the substrate (Fig. 2b). Some of the adenine rows are perfectly straight, while some others run in zigzag. The adenine rows decorate the whole surface of the one-atom high islands formed during the lifting of the reconstruction, whose dimensions seem to be related to the number of adsorbed adenine rows that can be accommodated on top of the islands.

Higher resolution images reveal that every single adenine strand is composed of brighter spots with a periodicity of approximately $3-3.5 \AA$ (Fig. 2c), in good agreement with the results obtained without potential control by Tao et al. [9]. Assuming that each spot corresponds to an 


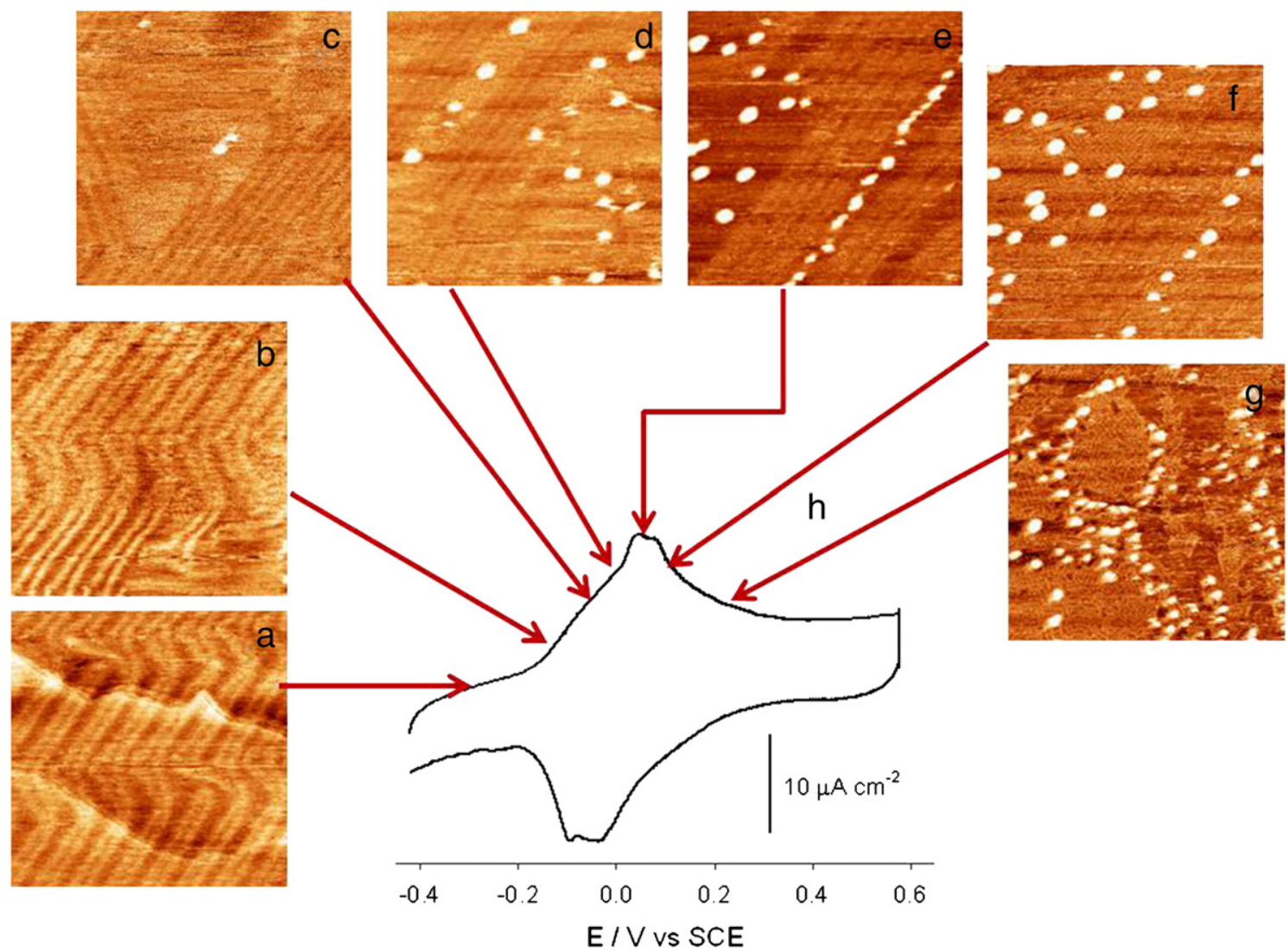

Fig. 1. Some $50 \times 50 \mathrm{~nm}^{2} \mathrm{EC}$-STM images of a $\mathrm{Au}(111)$ electrode in $0.1 \mathrm{M} \mathrm{H}_{2} \mathrm{SO}_{4}+1 \mathrm{mM}$ adenine. $I_{\mathrm{t}}=1 \mathrm{nA} ; U_{\mathrm{t}}=$ ranging from $0.15 \mathrm{~V}$ (tip positive) to $-0.35 \mathrm{~V}$ (tip negative), obtained sequentially at (a) $-0.2 \mathrm{~V}$ vs SCE, (b) $-0.09 \mathrm{~V}$ vs SCE, (c) $-0.04 \mathrm{~V}$ vs SCE, (d) $-0.01 \mathrm{~V}$ vs SCE, (e) $0.03 \mathrm{~V}$ vs SCE, (f) $0.06 \mathrm{~V}$ vs SCE and (g) $0.2 \mathrm{~V}$ vs SCE and (h) cyclic voltammogram at $20 \mathrm{mV} \mathrm{s}^{-1}$ in the same solution obtained in a separate electrochemical experiment.

adenine moiety, this excludes hydrogen bonding as the responsible for chain formation because any of the possible hydrogen bonds would involve distances between molecules between 5.8 and $7.8 \AA$ [9]. We suggest, hence, that the chains of adenine molecules are stabilized by $\pi$-staking interactions, a suggestion which is also compatible with the presence of both straight and zigzag adenine chains. $\pi-$ Staking electronic interactions have been described for purine basis in solution [15]. The distance between chains is of ca. $9 \pm 2 \AA$, which is too long for hydrogen bonding. We suggest, therefore, that the arrangement of adenine chains parallel to each other is governed by van der Waals interactions.

\section{Conclusions}

Chemisorption of adenine on $\mathrm{Au}(111)$ electrodes provokes the lifting of the reconstruction. The one-atom high gold islands formed by the $4 \%$ excess atoms ejected during the lifting of the reconstruction do not suffer Ostwald ripening nor coalesce with neighboring terrace steps due to the very low rate of atomic diffusion induced by the presence of chemisorbed adenine.

On the unreconstructed $\mathrm{Au}(111)$ surface, adenine molecules form chains that tend to follow the three main crystallographic directions of the substrate, although with frequent direction changes that impede
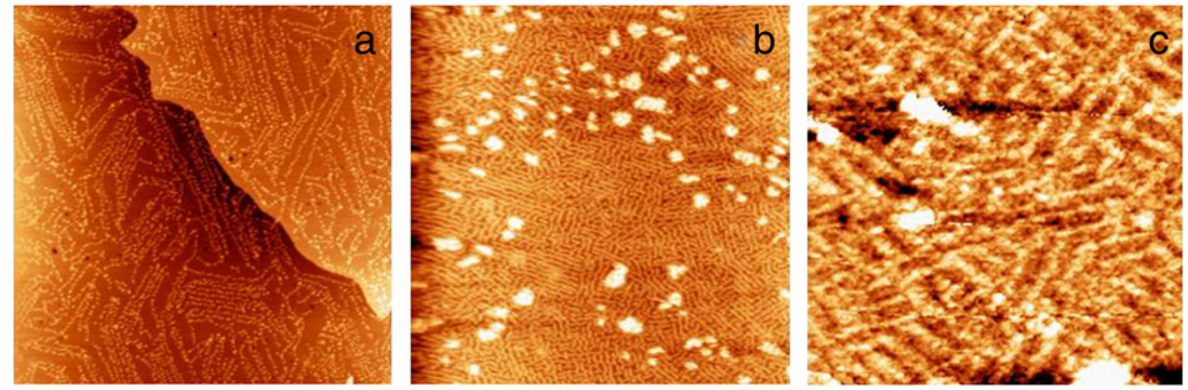

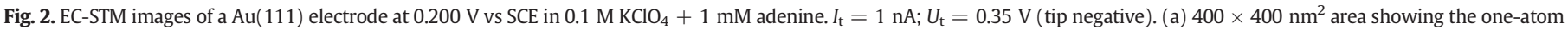

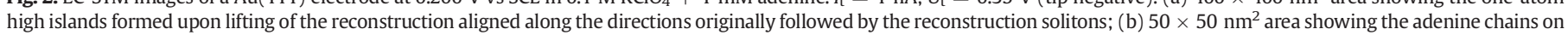
the unreconstructed $\mathrm{Au}(111)$ electrode; $(\mathrm{c})$ high-resolution $\left(11 \times 11 \mathrm{~nm}^{2}\right)$ EC-STM image of the adenine adlayer, $I_{\mathrm{t}}=0.6 \mathrm{nA}$. 
the formation of long chains. The chains align parallel to each other forming very small domains, resulting in an adlayer with a very short-range order. The intermolecular distances within chains, and the interchain distances suggest that chain formation is driven by $\pi$-stacking interactions, while parallel alignment of the chains is governed by van der Waals interactions.

This structure was observed in the whole $\mathrm{pH}$ range investigated (between 1 and 7), thus confirming that chemisorbed adenine is deprotonated even at $\mathrm{pHs}$ lower than the $\mathrm{p} K_{1}$ of adenine. STM observation of physisorbed protonated adenine, as recently detected by IR spectroscopy at low $\mathrm{pHs}$ and at potentials negative of the lifting of the reconstruction, was not possible, either because the physisorbed molecules are too mobile to be imaged by STM or because, due to their very weak interaction with the surface, they are swept away by the STM tip during imaging.

\section{Acknowledgements}

Financial support from the Spanish Ministry of Science and Technology (CTQ2009-07017 and CTQ2010-19823) and from the Junta de
Andalucia (PAI FQM202) is gratefully acknowledged. C. Vaz-Dominguez acknowledges a JAE Doc Fellowship from CSIC.

\section{References}

[1] C. Prado, F. Prieto, M. Rueda, J. Feliu, A. Aldaz, Electrochimica Acta 52 (2007) 3168.

[2] F. Prieto, M. Rueda, C. Prado, J. Feliu, A. Aldaz, Electrochimica Acta 55 (2010) 3301.

[3] A. Rodes, M. Rueda, F. Prieto, C. Prado, J.M. Feliu, A. Aldaz, Journal of Physical Chemistry C 113 (2009) 18784

[4] M. Rueda, F. Prieto, A. Rodes, J.M. Delgado, Electrochimica Acta 82 (2012) 534.

[5] Q. Chen, D.J. Frankel, N.V. Richardson, Langmuir 18 (2002) 3219.

[6] N.J. Tao, Z. Shi, Journal of Physical Chemistry 98 (1994) 1464

[7] R. Srinivasan, J.C. Murphy, N. Pattabiraman, Ultramicroscopy 42 (1992) 453

[8] R. Srinivasan, P. Galopan, Journal of Physical Chemistry 97 (1993) 8770.

[9] N.J. Tao, J.A. DeRose, S.M. Lindasy, Journal of Physical Chemistry 97 (1993) 910.

[10] D.M. Kolb, Progress in Surface Science 51 (1996) 109.

[11] C. Vaz-Domínguez, A. Aranzábal, A. Cuesta, Journal of Physical Chemistry Letters 1 (2010) 2059.

[12] Y. He, E. Borguet, Journal of Physical Chemistry C 115 (2011) 5726.

[13] T. Dretschkow, A.S. Dakkouri, T. Wandlowski, Langmuir 13 (1997) 2843.

[14] S. Wu, J. Lipkowski, O.M. Magnussen, B.M. Ocko, Th. Wandlowski, Journal of Electroanalytical Chemistry 446 (1998) 67.

[15] P.O.P. Ts'o, N.S. Kondo, R.K. Robins, A.D. Broom, Journal of the American Chemical Society 91 (1969) 5625 . 